\title{
Virally mediated inhibition of Bax in leukocytes promotes dissemination of murine cytomegalovirus
}

\author{
M Manzur ${ }^{1,2}$, P Fleming ${ }^{1,2}$, DCS Huang ${ }^{3}$, MA Degli-Esposti ${ }^{1,2,4}$ and CE Andoniou ${ }^{\star, 1,2,4}$
}

The evolutionary survival of viruses relies on their ability to disseminate infectious progeny to sites of transmission. The capacity to subvert apoptosis is thought to be crucial for ensuring efficient viral replication in permissive cells, but its role in viral dissemination in vivo has not been considered. We show here that the murine cytomegalovirus (MCMV) m38.5 protein specifically counters the action of Bax. As predicted from our biochemical data, the capacity of m38.5 to inhibit apoptosis is only apparent in cells unable to activate Bak. Deletion of $\mathrm{m} 38.5$ resulted in an attenuated growth of MCMV in vitro. In vivo replication of the $\Delta \mathrm{m} 38.5$ virus was not significantly impaired in visceral organs. However, $\mathrm{m} 38.5$ played a central role in protecting leukocytes from Bax-mediated apoptosis, thereby promoting viral dissemination to the salivary glands, the principal site of transmission. These results establish that in vivo MCMV replication induces the activation of Bax in leukocytes, but not other permissive cells, and that MCMV interferes with this process to attain maximum dissemination.

Cell Death and Differentiation (2009) 16, 312-320; doi:10.1038/cdd.2008.152; published online 24 October 2008

Apoptosis is considered to function as an innate defence mechanism against viral infection. This hypothesis has been strengthened by the fact that many viruses encode antiapoptotic proteins. ${ }^{1}$ However, the role of many viral antiapoptotic proteins in the context of in vivo infection has not been determined, but rather, has been inferred from the in vitro analysis of viral mutants.

$\mathrm{Bcl}-2$ proteins are the principal regulators of apoptosis in response to diverse stimuli. ${ }^{2}$ The $\mathrm{Bcl}-2$ family comprises opposing pro-apoptotic and antiapoptotic proteins that share one or more conserved regions, known as Bcl-2 homology (BH) domains. Pro-apoptotic family members can be further subdivided into the BH3-only group and the Bax/Bak subfamily; these proteins function in a coordinated manner to induce apoptosis.

The activity of the multidomain pro-apoptotic proteins, Bax and Bak, is essential for apoptosis to occur. ${ }^{3,4}$ The current paradigm suggests that Bax and Bak are restrained by prosurvival Bcl-2 proteins whose activity is, in turn, regulated by the $\mathrm{BH} 3$-only proteins. ${ }^{5-7}$ Although genetic studies in mice indicate that Bak and Bax are essentially redundant during development, ${ }^{3,8}$ some in vitro data suggest that apoptosis may be induced by the activation of either Bax or Bak. ${ }^{5,9}$ Furthermore, the inhibition of Bak is mediated by a subset of pro-survival $\mathrm{Bcl}-2$ proteins, whereas all pro-survival $\mathrm{Bcl}-2$ members appear to be capable of inhibiting Bax. ${ }^{5-7}$ These findings suggest that apoptosis may be initiated by the selective activation of either Bax or Bak. Whether in vivo apoptosis is initiated by the selective activation of either Bax or Bak is a critical, yet unresolved, question. Here, we addressed this issue using a viral infection model.

Human cytomegalovirus (HCMV) is an important human pathogen that causes significant mortality in immunocompromised individuals. HCMV encodes proteins with known antiapoptotic properties, ${ }^{10}$ whose activity is likely to be required to ensure that viral replication can occur within the host. However, given the strict species specificity of the CMV family, elucidating the in vivo role of these proteins is not possible. As the biology of murine CMV (MCMV) is similar to that of HCMV, MCMV has proved to be a useful model for studying the in vivo mechanisms utilised by CMVs to subvert host immune responses. We have previously demonstrated that MCMV-infected dendritic cells (DCs) are resistant to apoptosis induced by growth factor (GF) deprivation due to an inability to fully activate Bax. ${ }^{11}$ The viral mitochondrialocalised inhibitor of apoptosis (VMIA) protein encoded by exon 1 of UL37 in HCMV is a potent antiapoptotic protein that functions by sequestering Bax and keeping it inactive at the mitochondrial membrane. ${ }^{12,13}$ Recombinant HCMV mutants lacking VMIA produced using the AD169 strain do not replicate effectively. ${ }^{14}$ Surprisingly, the inactivation of VMIA in the Towne strain of HCMV had little effect on viral replication,

\footnotetext{
${ }^{1}$ Immunology and Virology Program, Centre for Ophthalmology and Visual Science, The University of Western Australia, Crawley, Western Australia, Australia; ${ }^{2} \mathrm{Centre}$ for Experimental Immunology, Lions Eye Institute, Nedlands, Western Australia, Australia and ${ }^{3}$ Molecular Genetics of Cancer Division, The Walter and Eliza Hall Institute of Medical Research, Parkville, Victoria, Australia

*Corresponding author: CE Andoniou, Centre for Experimental Immunology, Lions Eye Institute, 2 Verdun Street, Nedlands, Western Australia 6009, Australia.

Tel: + 6189381 0799; Fax: + 6189381 0700; E-mail: cadoniou@ cyllene.uwa.edu.au

${ }^{4}$ These authors contributed equally to this work

Keywords: apoptosis; cytomegalovirus; m38.5; Bax; viral dissemination

Abbreviations: BAC, bacterial artificial chromosome construct; BH, Bcl-2 homology; CMV, cytomegalovirus; DC, dendritic cell; FRT, FLP recombination target site; $\mathrm{HCMV}$, human cytomegalovirus; Kan ${ }^{\mathrm{R}}$, kanamycin resistance; KLH, keyhole limpet haemocyanin; MCMV, murine cytomegalovirus; MEF, murine embryonic fibroblast; $\mathrm{MOI}$, multiplicity of infection; ORF, open reading frame; PI, post-infection; PFU, plaque-forming unit; VMIA, viral mitochondria-localised inhibitor of apoptosis; WT, wildtype 
although cells infected with this mutant virus were more susceptible to apoptosis induced by proteasome inhibitors. ${ }^{15}$ It is unclear why the strain of HCMV used to produce VMIA mutants has such an impact on the phenotype observed. Both the AD169 and Towne strains of HCMV were developed as attenuated vaccine candidates by serial passage in fibroblasts and, consequently, do not have identical genomic structures. ${ }^{16}$ Therefore, the various $\triangle \mathrm{VMIA}$ mutants may have different phenotypes because of compounding mutations elsewhere in the viral genome. Alternatively, the strength of the apoptotic stimulus provided by HCMV infection may vary depending on the virus strain.

Together, the current data indicate that VMIA has antiapoptotic activity; however, the role of this protein in viral infection is unclear. The biochemical changes to Bax in MCMV-infected $D C^{11}$ mirror those induced by the overexpression of VMIA, suggesting that MCMV might encode a vMIA-like protein. Recently, m38.5 was identified as a potential VMIA orthologue ${ }^{15}$ prompting us to investigate the role of $\mathrm{m} 38.5$ in viral infection in vivo. Here, we have demonstrated that $\mathrm{m} 38.5$ is an antiapoptotic protein that functions by specifically inhibiting Bax. Surprisingly, m38.5 activity was not required for in vivo viral replication in the visceral organs. By contrast, MCMV replication in salivary glands, the major site of viral transmission, was attenuated by deletion of $\mathrm{m} 38.5$. We determined that $\mathrm{m} 38.5$ activity protects virally infected leukocytes from apoptosis, thereby ensuring efficient dissemination to the salivary glands. These results demonstrate that MCMV replication in vivo induces the activation of Bax in only a subset of permissive cells and that MCMV inhibits this process to ensure optimal viral dissemination.

\section{Results}

m38.5 is an antiapoptotic protein that binds Bax. Initially, we sought to determine if m38.5 possesses vMIA-like activity. Cos-7 cells were transiently transfected with a Flag-tagged m38.5 construct and immunoprecipitations performed using anti-Flag antibodies. Bax was found to coprecipitate with m38.5, but not with the MCMV-encoded M36 antiapoptotic protein (Figure 1a). The ability of m38.5 to associate with either Bax or Bak was then tested. HeLa cells were transiently transfected with a Flag-tagged m38.5 construct and immunoprecipitations performed. Although a clear association between $\mathrm{m} 38.5$ and Bax was detected, the closely related protein Bak did not co-precipitate with m38.5 (Figure 1b). The capacity of m38.5 to interact with murine Bax and Bak was then tested using mouse embryonic fibroblasts (MEFs). MEF cell lines derived from Bax or Bak knockout mice were transfected with a construct encoding Flag-tagged m38.5 and stable cell lines generated. Coimmunoprecipitation experiments using these cell lines confirmed that m38.5 is capable of associating with Bax, but not with Bak (Figure 1c). The capacity of m38.5 to inhibit apoptosis induced by the cytotoxic drug etoposide was then tested using wild-type (WT) MEFs or MEFs lacking Bax or Bak. Treatment of fibroblasts with etoposide causes Baxand Bak-mediated apoptosis, as either protein suffices to induce cell killing (Supplementary Figure 1). m38.5 specifically blocked etoposide-induced killing of cells lacking Bak (Figure 1d), but not of cells from the other genotypes tested. Thus, m38.5 selectively interacts with Bax to block its killing action.

Deletion of $\mathrm{m} 38.5$ impairs MCMV replication in vitro. To evaluate the role of $\mathrm{m} 38.5$ in viral infection, we engineered a mutant virus, termed $\Delta \mathrm{m} 38.5$, that lacks its expression. In constructing the $\Delta \mathrm{m} 38.5$ virus, one must consider that the m38.5 open reading frame (ORF) overlaps with M38 (Figure 2a). The m38.5 ORF was disrupted in the previously described K181 MCMV bacterial artificial chromosome construct (BAC) pARK25 ${ }^{17}$ by homologous recombination. This process involved replacing a portion of the m38.5 ORF (nucleotides 52316-52240) with the kanamycin resistance $\left(\mathrm{Kan}^{\mathrm{R}}\right)$ gene flanked by FLP recombination target (FRT) sites. Removal of the $\mathrm{Kan}^{\mathrm{R}}$ cassette was achieved by FLP recombination between the FRT sites. Sequencing of the resulting mutant indicated that the FRT remnant introduces several in-frame stop codons (Figure 2b) and a frameshift mutation downstream of nucleotide 52240 . The result of these changes is that the m38.5 ORF is now only capable of producing a fusion protein consisting of the first 17 amino acids of m38.5 and 14 amino acids encoded by the FRT remnant. Northern blot analysis was performed on the $\Delta$ m38.5 mutant to ensure that the surrounding ORFs were not affected by the mutagenesis procedure. The ORFs in the vicinity of m38.5 use the same poly-A site; hence, probes used to detect the various RNA transcripts in this region detect multiple RNA species (Figure 2c). Transcripts for both $\mathrm{m} 39$ and $\mathrm{m} 40$ were detected in similar amounts in both the $\Delta \mathrm{m} 38.5$ virus and WT MCMV (Figure 2d), indicating that the mutagenesis procedure had not affected the transcription of these ORFs. Owing to the small difference in their size, the m38.5 and M38 transcripts could not be distinguished in the northern blot analysis (Figure 2d). Sequencing of the $\Delta$ m38.5 virus verified that the area surrounding the transcriptional start site of M38 had not been affected. To confirm this, $5^{\prime}$ rapid amplification of cDNA ends (RACE) products from cells infected with the $\Delta \mathrm{m} 38.5$ virus were sequenced. We found that M38 transcripts were present in cells infected with the $\Delta \mathrm{m} 38.5$ and that these transcripts encode the predicted sequence (data not shown). A revertant virus, m38.5 Rev, was also generated by re-inserting the full-length m38.5 ORF into the $\Delta \mathrm{m} 38.5$ virus by homologous recombination. Confirmation that the m38.5 protein was not produced by the $\Delta$ m38.5 mutant virus was obtained by immunoblot analysis (Figure 2e). Probing with an anti-IE1-specific monoclonal antibody confirmed equivalent levels of infection in all samples (Figure 2e).

The growth characteristics of the $\Delta \mathrm{m} 38.5$ virus were assessed in a range of permissive cell types. Cells were infected with WT, $\Delta \mathrm{m} 38.5$ virus or m38.5 Rev virus at a multiplicity of infection (MOI) of 0.02 and viral replication at various times post-infection $(\mathrm{PI})$ determined by plaque assay. The absence of m38.5 did not have an appreciable impact on viral replication during the first $2-3$ days $\mathrm{PI}$, but after this point, the replication of the $\Delta \mathrm{m} 38.5$ mutant virus was consistently 


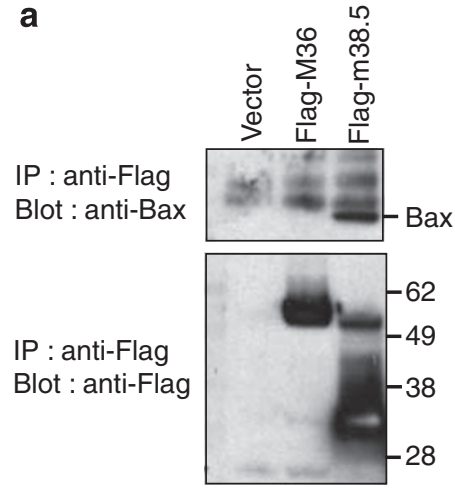

C

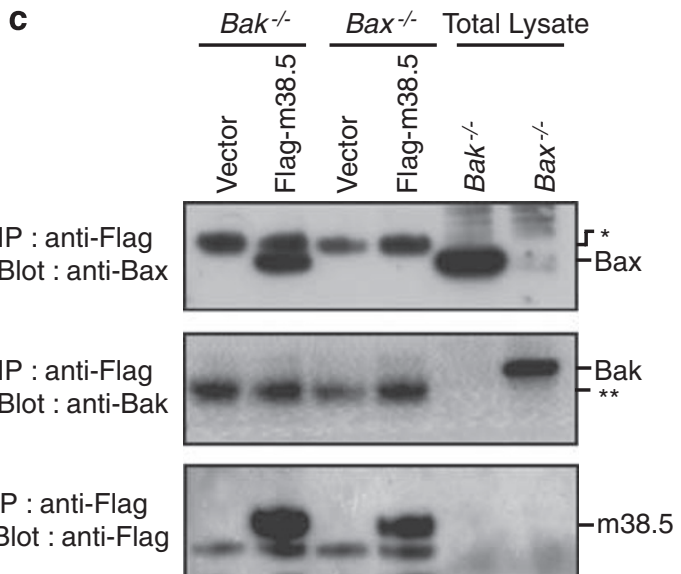

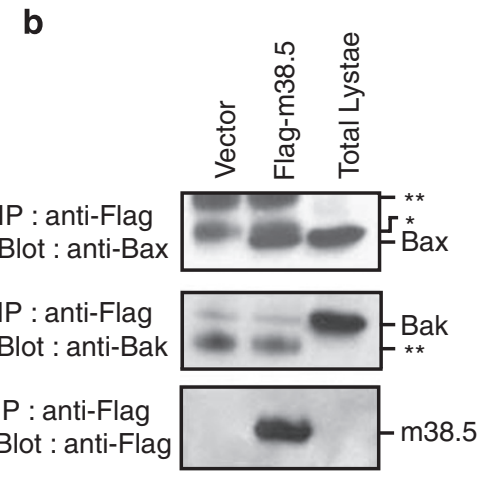

d

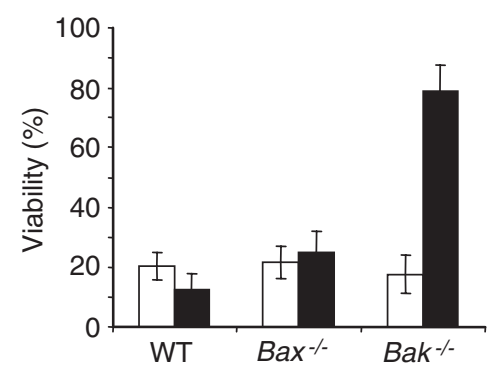

Figure $1 \mathrm{~m} 38.5$ inhibits apoptosis in a Bax-specific manner. (a) Cos cells were transiently transfected with Flag-tagged constructs encoding m38.5, M36 or empty vector. Cells were lysed in the presence of $2 \%$ CHAPS, Flag-tagged proteins immunoprecipitated and bound proteins analysed by immunoblot using the indicated antibodies. (b) HeLa cells were transfected with Flag-tagged $\mathrm{m} 38.5$ construct or empty vector. Flag-tagged proteins were immunoprecipitated and bound proteins analysed by immunoblot using the indicated antibodies. ${ }^{*}$ Background band; ${ }^{*} \mathrm{IgG}$ light chain. (c) Lysates were prepared from the indicated MEF cell lines expressing Flag-tagged m38.5 and Flagtagged proteins immunoprecipitated. Bound proteins were analysed by immunoblotting with the indicated antibodies. *Background band; ** $\mathrm{gG}$ light chain. (d) MEFs derived from WT, bax- or bak-deficient mice were infected with a retrovirus encoding m38.5 and GFP (filled columns) or GFP alone (open columns) and incubated for $48 \mathrm{~h}$. Cells were then treated with etoposide and viability determined $16 \mathrm{~h}$ later $(n=8)$

attenuated (Figure 3a). Importantly, the m38.5 Rev virus replicated as well as the WT virus in all cells tested (Figure 3a). Thus, the absence of m38.5 expression impairs MCMV replication in vitro. The impaired growth phenotype of the $\Delta \mathrm{m} 38.5$ mutant could be attributed to its inability to inhibit Bax and, possibly, Bak. Importantly, when compared with the parental WT virus, growth of the $\Delta \mathrm{m} 38.5$ virus was unaffected in cells lacking Bax, but was impaired in both WT and bak ${ }^{-/-}$ cells (Figure $3 b$ ). Hence, m38.5 promotes viral replication by preventing the activation of Bax, but not Bak.

CMVs are capable of replicating in a broad range of cell types. Next, we determined if the ability of MCMV to replicate in leukocytes was dependent on the expression of m38.5. The $\Delta \mathrm{m} 38.5$ virus displayed a pronounced growth defect in DCs or macrophage cell lines (Figure 4a). We have previously shown that following MCMV infection, DCs become resistant to apoptosis, and this coincides with a redistribution of Bax from the cytosol to mitochondria. ${ }^{11}$ Subcellular fractionation of DC infected with the $\Delta$ m38.5 virus indicated that Bax was predominately localised to the cytoplasm (Figure 4b). Furthermore, unlike DCs infected with the WT virus, DCs infected with the $\Delta$ m38.5 mutant were susceptible to apoptosis induced by GF withdrawal (Figure 4c). Taken together, our data establish that m38.5 enhances MCMV replication by keeping Bax in an inert state on the mitochondria.

MCMV infection inhibits the activation of Bak and Bax. Growth of the $\Delta \mathrm{m} 38.5$ virus was equivalent to that of the WT virus in Bax-null fibroblasts, but attenuated in Baknull fibroblasts (Figure $3 b$ ). An implication of this result is that replication of MCMV in fibroblasts must induce apoptosis exclusively through Bax. Alternatively, MCMV infection may trigger the activation of both Bax and Bak, and MCMV encodes a yet-to-be identified inhibitor of Bak. To distinguish between these possibilities, we infected WT fibroblasts or fibroblasts lacking Bax or Bak with either WT MCMV or the $\Delta$ m38.5 mutant virus, using an $\mathrm{MOI}$ of 3 to ensure that all cells within the culture were infected. Virally infected cells were then treated with etoposide to test their susceptibility to apoptosis. As mentioned earlier, in fibroblasts, etoposide treatment induces the activation of both Bax and Bak, with either sufficient to induce apoptosis. All fibroblast lines 
a

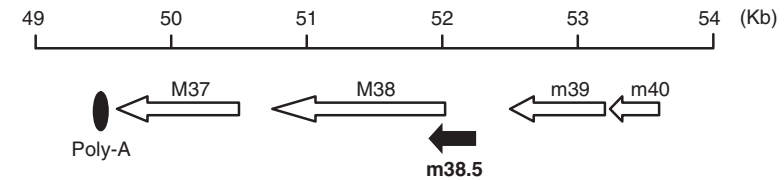

b

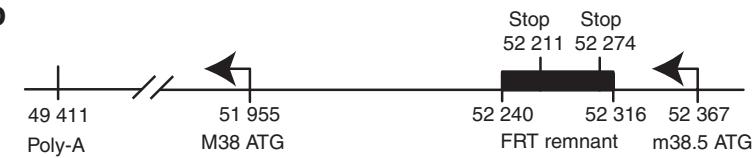

C

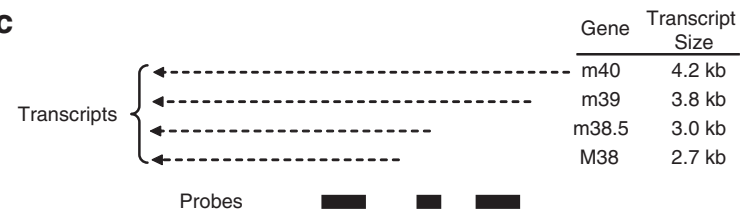

d
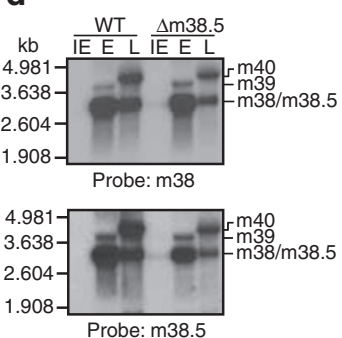

e

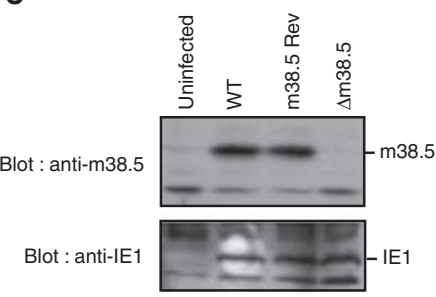

Probe: $\mathrm{m} 39$

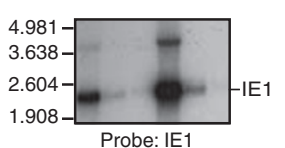

Figure 2 Genomic arrangement and analysis of $\Delta \mathrm{m} 38.5$. (a) The genomic region of MCMV encompassing m38.5 (solid arrow) and adjoining ORFs (open arrows) is shown. Direction of gene transcription is denoted by the direction of the arrows. Location of the common polyadenylation site used by all genes in this region is denoted by the filled circle. (b) Sequencing of $5^{\prime}$ RACE products and of viral DNA was performed and the data used to generate a map of the genomic structure of the $\Delta \mathrm{m} 38.5$ mutant virus. The FLP remnant retained from the mutagenesis procedure causes a frameshift downstream of nucleotide 52316 and introduces premature stop codons. (c) Size of the various gene transcripts in the region is shown. The probes used for northern analysis and their respective binding sites are shown (filled boxes). (d) RNA was isolated from fibroblasts infected with WT MCMV-K181 Perth or the $\Delta$ m38.5 mutant at immediate early $(\mathrm{IE})$, early $(\mathrm{E})$ and late $(\mathrm{L})$ times after infection. Total RNA was separated on a denaturing agarose gel, blotted onto PVDF membrane and hybridised with DNA probes. (e) Total cell lysates were prepared from fibroblasts infected with the indicated viruses $24 \mathrm{~h} \mathrm{PI}$. Immunoblot analysis was performed using hyperimmune serum from a rat injected with an m38.5 peptide linked to KLH or an anti-IE1 monoclonal antibody

infected with WT MCMV were resistant to apoptosis induced by etoposide treatment (Figure 5), suggesting that MCMV can prevent the activation of both Bax and Bak. WT or bak ${ }^{-1-}$ fibroblasts infected with the $\Delta \mathrm{m} 38.5$ virus were sensitive to etoposide-induced apoptosis, with only a few viable cells

remaining $18 \mathrm{~h}$ after the addition of the drug (Figure 5). This result is consistent with the hypothesis that cells infected with the $\Delta \mathrm{m} 38.5$ virus lack the ability to prevent the activation of Bax. By contrast, Bax-deficient fibroblasts infected with the $\Delta$ m38.5 mutant did not undergo apoptosis after etoposide treatment (Figure 5). Therefore, in addition to encoding a Bax-specific antiapoptotic protein, MCMV must also possess a strategy to inhibit Bak activation.

Viral load within the salivary gland is dependent on m38.5 activity. As growth of the $\Delta$ m38.5 mutant virus was attenuated in vitro, we anticipated that viral replication would be similarly compromised in vivo. The capacity of the $\Delta \mathrm{m} 38.5$ virus to replicate in vivo was assessed by comparing viral titres in the target organs of BALB/c mice infected with either WT or the $\Delta$ m38.5 mutant virus. Mice were infected with either WT or $\Delta$ m38.5 virus, and viral titres in target organs determined by plaque assay at various times PI. Surprisingly, viral titres in the spleen, liver and lungs of mice infected with the $\Delta \mathrm{m} 38.5$ virus did not differ significantly from those observed in the organs of mice infected with WT MCMV (Figure 6a). By contrast, viral titres in the salivary glands were 10 - to 100 -fold lower for the $\Delta$ m38.5 mutant compared with the WT virus (Figure 6b). To ensure that the impaired replication observed in salivary glands was due to the loss of m38.5 activity, the growth of the m38.5 Rev virus was assessed in vivo. Titres of $\mathrm{m} 38.5 \mathrm{Rev}$ in the salivary glands were similar to those of the WT virus, whereas significantly reduced viral titres were observed in salivary glands of mice infected with the $\Delta \mathrm{m} 38.5$ virus (Figure $6 \mathrm{c}$ ). Therefore, although m38.5 is not required for efficient viral replication in all permissive cell types in vivo, the protein exerts a selective effect that promotes MCMV replication in salivary glands.

m38.5 enhances MCMV replication in the blood. The reduced salivary gland titres seen after infection with the $\Delta \mathrm{m} 38.5$ virus may indicate a requirement for m38.5 activity for the survival of acinar cells, the predominant target of MCMV in this tissue. ${ }^{18}$ Alternatively, dissemination of the $\Delta \mathrm{m} 38.5$ virus to salivary glands could be impaired. MCMV dissemination is mediated by myeloid progenitors that enter the circulation after being mobilised from the bone marrow. ${ }^{19-21}$ As attenuation of $\Delta \mathrm{m} 38.5$ growth in vitro was most pronounced in cells of myeloid origin (Figure 4a), we investigated whether the deletion of m38.5 had any impact on viral load in the blood. Blood samples from mice infected with WT or $\Delta \mathrm{m} 38.5$ virus were collected and the number of infected cells in the circulation determined by infectious centre assay. At day 5 after infection, the time when MCMV titres in the blood peak, ${ }^{20}$ fewer cells harbouring infectious virus were detected in the blood of mice infected with the $\Delta \mathrm{m} 38.5$ mutant (Figure 7a). This finding suggests that m38.5 may be necessary for viral replication to proceed efficiently in leukocytes, thereby ensuring maximal viral trafficking to the salivary glands.

If replication of the m38.5 mutant virus within leukocytes is impaired, this should be apparent at other sites. This possibility was investigated by examining viral replication within the spleen. MCMV replication in the spleen occurs in 

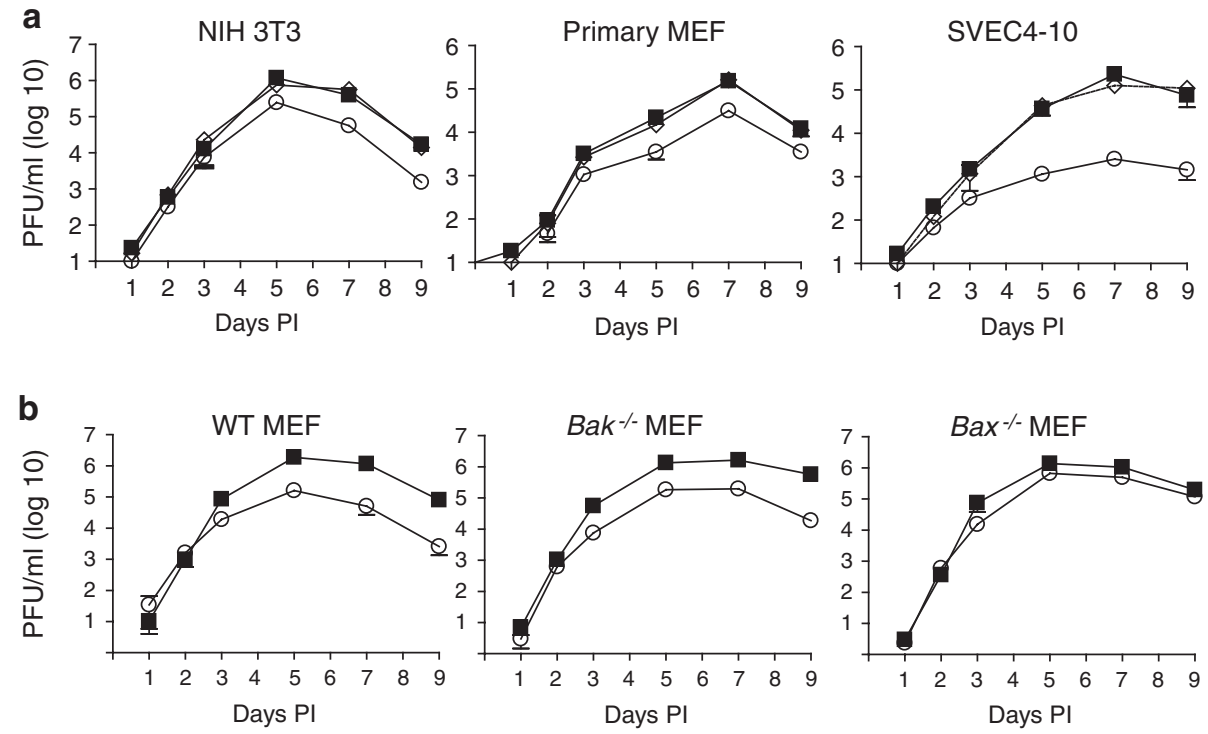

Figure 3 In vitro growth of the $\Delta \mathrm{m} 38.5$ virus. (a) Primary MEFs, NIH 3 T3 fibroblasts and SVEC4-10 epithelial cells were infected with either WT (filled squares), $\Delta \mathrm{m} 38.5$ (open circles) or $\mathrm{m} 38.5 \mathrm{Rev}$ virus (open diamonds) at an $\mathrm{MOI}$ of 0.02 . At the indicated times after infection, total viral yield was determined $(n=3)$. (b) Fibroblast cell lines from WT, bax- or bak-deficient mice were generated from E13-E14.5 embryos using SV40 large T antigen. ${ }^{5}$ The various cell lines were infected at an MOI of 0.02 with either WT MCMV (filled squares) or the $\Delta$ m38.5 mutant (open circles) and viral yield (PFU) determined at the indicated times after infection $(n=3)$

a

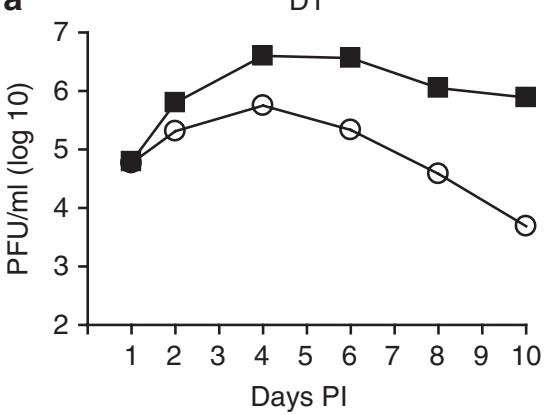

b

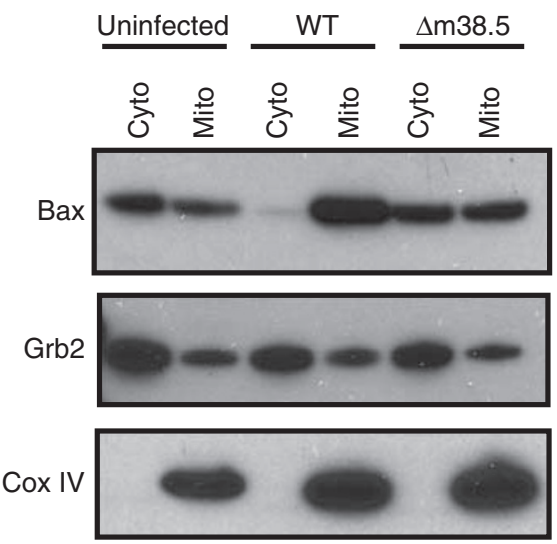

IC-21

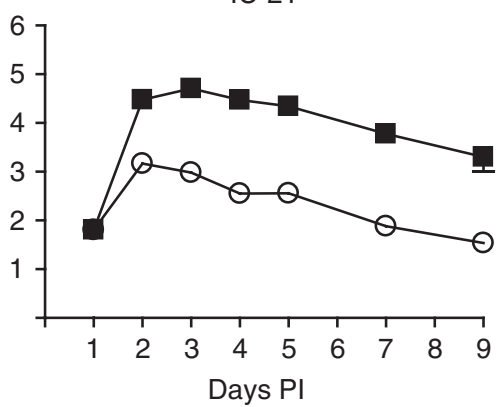

C

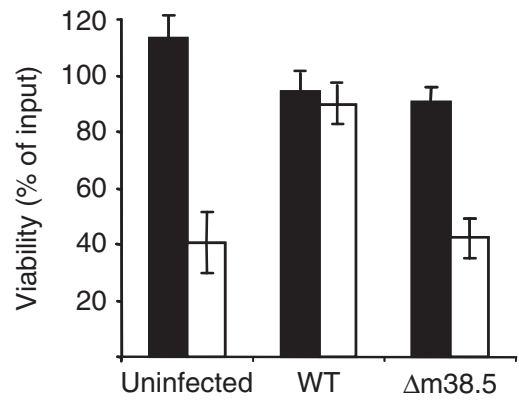

Figure $4 \mathrm{~m} 38.5$ promotes viral replication by binding Bax at the mitochondria. (a) D1 DCs or IC-21 macrophages were infected with WT (filled squares) or $\Delta \mathrm{m} 38.5$ virus (open circles) at an MOI of 3 and the viral yield determined ( $n=8$ for D1 and $n=3$ for IC-21). (b) D1 DCs were infected with WT or $\Delta \mathrm{m38.5}$ virus (MOI $=3$ ) and 4 days after infection, cytoplasmic (Cyto) and mitochondrial (Mito) fractions prepared and immunoblotted with the indicated antibodies. (c) D1 DCs were infected with WT or $\Delta$ m38.5 virus $(\mathrm{MOI}=3)$ and 4 days after infection the cells were plated in complete medium (filled columns) or medium lacking GF (open columns) and viability determined $18 \mathrm{~h}$ later $(n=10)$

various cell types, including endothelial cells ${ }^{22}$ and leukocytes, such as macrophages ${ }^{19}$ and DCs. ${ }^{23} \mathrm{~A}$ specific defect in viral replication within leukocytes might not have been observed in our initial analysis as we determined viral loads in the whole organ. Therefore, spleens from mice infected with either WT or $\Delta \mathrm{m} 38.5$ viruses were removed at day $4 \mathrm{PI}$ and 

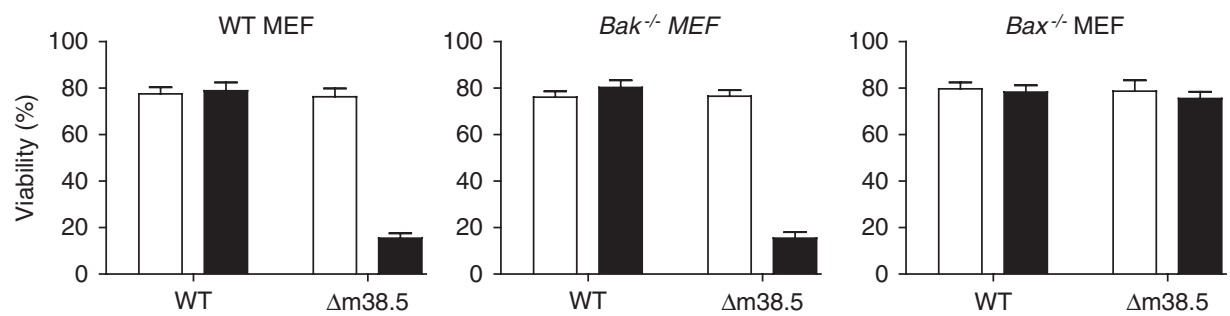

Figure $5 \mathrm{MCMV}$ encodes inhibitors for both Bax and Bak. The indicated MEF cell lines were infected with either WT or $\Delta \mathrm{m} 38.5$ virus $(\mathrm{MOI}=3)$ and incubated for $18 \mathrm{~h}$. Etoposide (filled columns) or vehicle (open columns) was added to the various cultures and viability determined $24 \mathrm{~h}$ later $(n=7)$
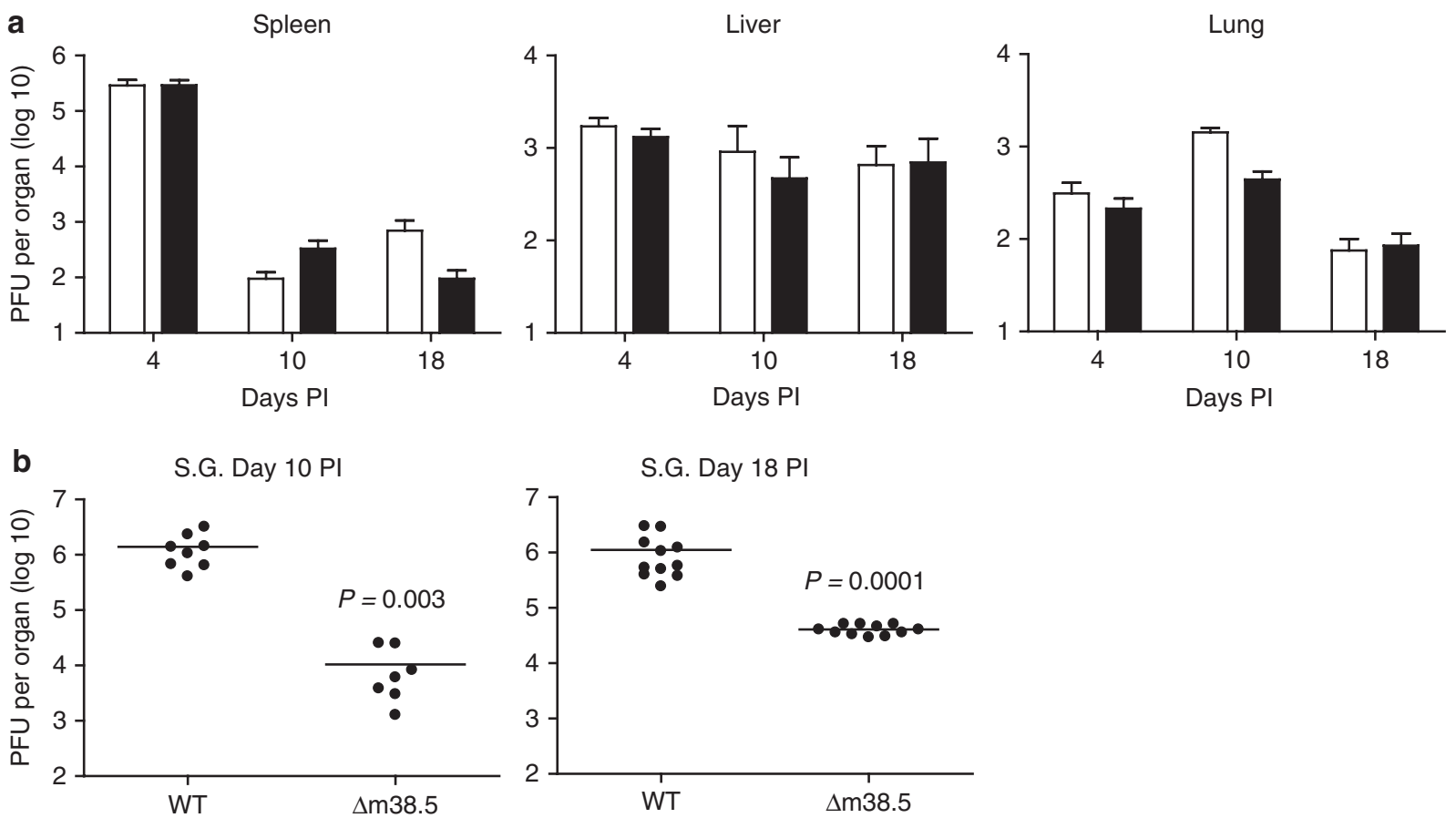

C

S.G. Day 10 PI
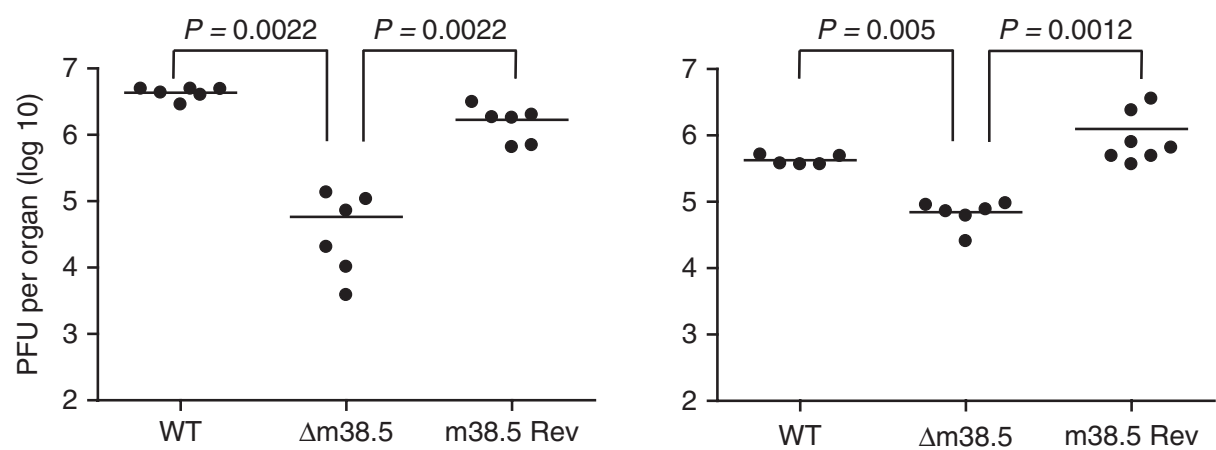

Figure 6 Loss of m38.5 reduces viral load in the salivary gland. (a) Mice were infected with WT MCMV (open columns) or $\Delta$ m38.5 virus (filled columns), organs were removed at various times after infection and viral load per organ determined by plaque assay. Viral titres were quantified in three separate experiments and the data pooled, mean \pm S.D. of seven to nine mice per group is plotted. No statistically significant differences were observed at any time point. (b) Viral load in the salivary gland of mice infected with either WT or $\Delta \mathrm{m} 38.5$ virus at the indicated times after infection was determined by plaque assay. Data from four experiments have been pooled. (c) Mice were infected with the indicated viruses and viral titres in the salivary glands determined at the time points indicated. Data are pooled from two experiments

half the organ processed to purify leukocytes while excluding endothelial cells. FACS analysis indicated that greater than $95 \%$ of the cells isolated using this method were positive for the leukocyte antigen CD45 (data not shown). The leukocytes were lysed and viral load determined by plaque assay. A significant reduction in viral replication within splenic leukocytes was observed in mice infected with the $\Delta$ m38.5 virus (Figure $7 \mathrm{~b}$, right panel). The remaining half of the spleen was 
a

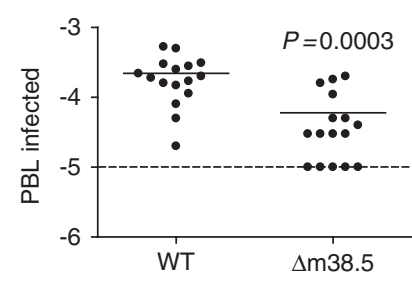

b

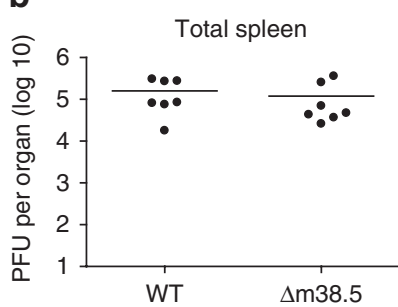

Leukocytes

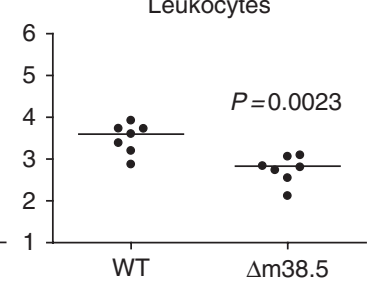

Figure 7 The role of m38.5 is specific for leukocytes. (a) Whole blood was isolated from infected mice, red blood cells removed by hypotonic lysis and the proportion of infected cells determined by infectious centre assay. (b) Spleens from mice infected with WT or $\Delta \mathrm{m} 38.5$ virus were removed and total viral burden was determined using half the spleen (left panel). The remainder of the spleen was used to determine viral load within leukocytes purified as described (right panel)

used to determine total viral load, and as with our earlier results no difference was observed in viral titres from the whole organ (Figure 6a, left panel). Together, these results establish that m38.5 activity is required to enhance viral replication within leukocytes. By contrast, deletion of $\mathrm{m} 38.5$ had no effect on the ability of MCMV to replicate in other permissive cell types, such as endothelial cells, in vivo.

\section{Discussion}

In this study, we have examined the role of m38.5 in the pathogenesis of MCMV and have established that it is a Baxspecific inhibitor of apoptosis. In vivo, m38.5 expression enhanced viral replication within leukocytes, thereby promoting dissemination of MCMV to the salivary glands. Our data indicate that viral interference with apoptosis not only improves viral replication, but is also required to promote efficient viral dissemination.

Given the central role played by the Bax/Bak proteins in promoting cellular destruction, it is not surprising that a number of viruses encode proteins capable of preventing their activation. For example, adenovirus, myxoma virus and Epstein-Barr virus encode antiapoptotic proteins that function by preventing the activation of both Bax and Bak. ${ }^{24-28}$ As the size of viral genomes is limited, encoding a single protein capable of inhibiting both Bax and Bak may provide the pathogen with a survival advantage with limited burden on the viral genome. This, however, does not seem to be the case for MCMV, as we provide evidence that Bak activation in MCMV infection is inhibited independently of m38.5-mediated activities. These findings indicate that MCMV encodes specific inhibitors of Bax (m38.5) and Bak, with the latter yet to be identified. Our in vitro data are consistent with recent publications demonstrating that $\mathrm{m} 38.5$ is a Bax-specific antiapoptotic protein. ${ }^{29-31}$ Interestingly, the related VMIA protein encoded by HCMV is capable of inhibiting both Bax and Bak. ${ }^{30,32}$ Why MCMV encodes specific and separate inhibitors of Bax and Bak, when many viruses, including the closely related $\mathrm{HCMV}$, encode one antiapoptotic protein able to inactivate both pro-death mediators remains to be clarified. In this respect, it is worth noting that although Bax/Bak are essential for apoptosis, the role of these proteins in $\mathrm{Ca}^{2+}$ homoeostasis and mitochondrial morphogenesis in healthy cells has also been established. ${ }^{32,33}$ Indeed, the expression of VMIA is sufficient to disrupt mitochondrial networks and induce $\mathrm{Ca}^{2+}$ mobilisation from the endoplasmic reticulum. ${ }^{34,35}$ Consistent with the inability of m38.5 to bind Bak, expression of m38.5 does not interfere with mitochondrial morphology unless Bak is absent. ${ }^{30}$ Therefore, cell lineagespecific expression of Bax or Bak viral inhibitors may allow MCMV to inhibit apoptosis, while minimising the impact on other cellular processes required for viral replication.

A central finding of this study is that rather than having a global impact on viral replication, deletion of $m 38.5$ resulted in a reduction in viral load specifically in salivary glands. Salivary gland tropism is an important feature of CMV infection, with shedding of the virus into the saliva serving as the principal source of horizontal transmission. ${ }^{36}$ Reduced salivary gland titres were the result of an impaired ability of the $\Delta \mathrm{m} 38.5$ mutant to replicate in leukocytes, cells known to be required for the dissemination of MCMV to the salivary glands. The finding that m38.5 enhanced viral replication in leukocytes, but not other permissive cell types in vivo, could be reconciled by the absence of Bax expression in cells or tissues where we did not observe a difference in the ability of the $\Delta \mathrm{m} 38.5$ mutant viruses to replicate. However, Bax has a broad expression pattern in vivo and, in particular, is expressed in both the spleen and lungs. ${ }^{37}$ Hence, the capacity of the $\Delta \mathrm{m} 38.5$ virus to replicate normally in cells other than leukocytes cannot simply be attributed to a lack of Bax, but rather, suggests that MCMV infection does not lead to activation of Bax in these cells during in vivo infection.

In vivo replication of the $\Delta \mathrm{m} 38.5$ mutant was attenuated specifically in leukocytes, but in vitro replication of the $\Delta \mathrm{m} 38.5$ virus was impaired in a variety of cell types. The broader defect observed in vitro may be the result of cells being subjected to stress while monitoring viral growth over a period of 9 days. During this time, nutrients and GFs in the culture medium are gradually depleted, and this has the potential to act as an apoptotic stimulus. Our data indicate that cells infected with the $\Delta \mathrm{m} 38.5$ virus are less resistant to apoptotic stimuli, such as GF deprivation or etoposide treatment, than those infected with WT virus. Thus, growth of the $\Delta$ m38.5 virus may be attenuated in a wide range of cell types in vitro due to the presence of additional apoptotic stimuli that are not at play during in vivo infection. Support for this hypothesis comes from the observation that the in vitro growth of a $\Delta \mathrm{m} 38.5$ virus is significantly enhanced by changing the culture medium daily. ${ }^{29}$ The in vitro data clearly define m38.5 as a specific inhibitor of Bax. In vivo, the capacity to inhibit Bax is required to promote dissemination of MCMV.

Activation of Bax and/or Bak is a prerequisite for apoptosis to proceed in response to many stimuli., ${ }^{3,4}$ Bax and Bak function in a redundant fashion during development; however, 
in vitro studies have suggested that Bax and Bak may be independently activated, and the ability of some BH3-only proteins to selectively antagonise the function of pro-survival $\mathrm{Bcl}-2$ proteins has been noted. ${ }^{38}$ Our data indicate that MCMV replication in vivo induces the activation of Bax in leukocytes, but not in other permissive cell types. It is unclear why MCMV infection in vivo results in activation of Bax in leukocytes, but this selectivity is most likely due to leukocytes expressing a different combination of pro- and antiapoptotic $\mathrm{Bcl}-2$ proteins than that expressed by other MCMV-permissive cells.

In summary, we have demonstrated that m38.5 inhibits Bax activation in leukocytes. Inhibition of Bax by MCMV in leukocytes was required to achieve optimal dissemination to the salivary gland, the main site of viral transmission. We also provide evidence that MCMV inhibits the activation of Bak and that this function is independent of m38.5. How MCMV inhibits Bak is the subject of our on-going studies. A detailed understanding of how CMVs inhibit apoptosis might be relevant to the development of novel interventions against viral infection.

\section{Materials and Methods}

Cell lines and reagents. Culture conditions for cell lines and methods for the purification of viral stocks have been described previously. ${ }^{5,11,23} \mathrm{Cos}-7$ or HeLa cells were transfected with pcDNA3 encoding Flag-m38.5, Flag-M36 or vector only using Fugene-6 reagent (Roche) and total cell lysates prepared $48 \mathrm{~h}$ later. Similarly, MEFs were transfected with pcDNA3 encoding Flag-m38.5 or vector only using Fugene-6 reagent and stable cell lines generated by puromycin selection. Retroviral-mediated transfection was performed as described. ${ }^{5}$ Immunoprecipitation analysis was performed on cell lysates prepared using CHAPS detergent according to published methods. ${ }^{11}$ Induction of apoptosis in DC and subcellular fractionation were performed as described. ${ }^{11}$ Apoptosis in MEF was induced by the addition of $100 \mu \mathrm{M}$ etoposide (Sigma) and viable cells enumerated by trypan blue exclusion. Hyperimmune serum against m38.5 was produced by injecting a rat with a peptide comprising amino acids $40-53$ of m38.5 linked to keyhole limpet haemocyanin. TiterMax Classic adjuvant (Sigma Chemicals) was mixed with $100 \mu \mathrm{g}$ of the peptide ( $1: 1$ ratio) and injected I.P. The rat was boosted 28 days after the primary injection. Serum was purified from whole blood 10 days after the boost and used for immunoblot analysis.

Analysis of viral growth. The $\triangle \mathrm{m} 38.5$ mutant virus was constructed using the MCMV-K181 Perth BAC. ${ }^{17}$ Inbred BALB/c mice at 8 weeks of age were obtained from the Animal Resources Centre (Perth, Western Australia) and maintained in specific pathogen-free conditions at the Animal Services Facility of the University of Western Australia. Mice were injected intraperitoneally with $1 \times 10^{4}$ plaque-forming units (PFUs) of salivary gland-propagated virus stock of WT BAC-derived MCMV$\mathrm{K} 181$ or $\Delta \mathrm{m} 38.5$ virus diluted in phosphate-buffered saline containing $0.5 \% \mathrm{FBS}$. At various time points after infection, mice were killed and organs removed for analysis. Viral titres were quantified by plaque assay on monolayers of permissive cells. ${ }^{23}$ Viraemia in the blood was assessed by isolating blood by heart puncture and determining the number of infected cells by culture on permissive cells. ${ }^{20}$ Leukocytes in the spleen were isolated as described. ${ }^{22}$ FACS analysis indicated that $>95 \%$ of the cells isolated using this procedure were leukocytes. All animal experimentations were performed with the approval of the Animal Ethics and Experimentation Committee of the University of Western Australia and according to the guidelines of the National Health and Medical Research Council of Australia. For in vitro analysis of viral replication, tissue culture stocks of WT virus, $\Delta \mathrm{m} 38.5$ or m38.5 Rev were produced by infecting NIH 3T3 fibroblasts with the respective salivary gland-propagated viruses. Viral stocks were harvested and purified as described earlier. ${ }^{39}$

Generation of $\mathbf{m} 38.5$ constructs. The m38.5 ORF was amplified by PCR from DNA isolated from the MCMV-K181 Perth viral strain and cloned into the EcoR1 site of the pcDNA3Flag expression vector (Invitrogen) or into the pMIG retroviral vector. DNA sequencing was employed to ensure the fidelity of the PCR product. Details of all oligonucleotides and plasmids are available from the authors.

Viral mutagenesis. A recombinant MCMV genome containing a disruption in the m38.5 ORF was constructed in Escherichia coli. The pSLFRTKn plasmid was used as a template ${ }^{40}$ to generate a PCR product containing the Kan ${ }^{\mathbb{R}}$ gene flanked by $50 \mathrm{bp}$ of homology to the m38.5 target sequence and $34 \mathrm{bp} \mathrm{FRT} \mathrm{site} \mathrm{in} \mathrm{the} \mathrm{same}$ orientation. Following purification, the PCR fragment was transformed into the DH10 $\beta$ strain of $E$. coli carrying the previously described K181 MCMV BAC pARK2 $5{ }^{17}$ Deletion of the target sequence within the m38.5 ORF was achieved by homologous recombination, mediated by the $\lambda$ red recombinase-encoding plasmid pKD46. ${ }^{40}$ Transformants were selected at $30^{\circ} \mathrm{C}$ on LB agar plates containing chloramphenicol $(25 \mu \mathrm{g} / \mathrm{ml})$ to select for the ARK25 bacmid and kanamycin $(25 \mu \mathrm{g} /$ $\mathrm{ml}$ ). Kan ${ }^{\mathbb{R}}$ clones were screened by PCR to confirm the insertion of the Kan ${ }^{\circledR}$ gene. Removal of the $\operatorname{Kan}^{\mathbb{R}}$ cassette was achieved by transforming $\operatorname{Kan}^{\mathbb{R}}$ mutants with pCP20, which directs the excision of the Kan ${ }^{\circledR}$ cassette through FLP recombination between the FRT sites. ${ }^{40}$ Loss of the Kan ${ }^{\circledR}$ gene was assessed by replica plating onto LB agar plates containing either chloramphenicol alone or chloramphenicol and kanamycin. Excision of the Kan ${ }^{\circledR}$ gene by FLP recombination leaves an FRT remnant within the m38.5 ORF. Sequencing of $\Delta \mathrm{m} 38.5$ indicated that the FRT remnant introduced a frameshift mutation in the m38.5 ORF (Figure $2 b$ ).

Reconstitution of virus progeny from MCMV BAC plasmids. MEFs derived from $\mathrm{Bax}^{-1-} \mathrm{Bak}^{-/-}$mice were seeded into a sixwell tray at a density of $1 \times 10^{5}$ cells per well before transfection. pARK25 $\Delta$ m38.5 DNA was purified using the NucleoBond nucleic acid purification kit (BD Biosciences) according to the manufacturer's instructions. MEFs were transfected with viral DNA $(1 \mu \mathrm{g})$ using FuGENE-6 (Roche) and cultured until plaques developed. Successful transfection and reconstitution of the virus was monitored by GFP expression driven by the EGFP gene within the BAC backbone of ARK25. Once the monolayer exhibited maximal cytopathic effect, viral supernatants were collected. The BAC backbone was removed from the viral genome by serial passage of the virus on MEF monolayers and monitoring for the loss of GFP expression. The BAC cassette is flanked by a 249 -bp MCMV sequences repeat. ${ }^{17}$ When transfected into eukaryotic cells, homologous recombination between the repeat MCMV sequences results in the excision of the BAC cassette. The reconstituted virus was denoted MCMV $\triangle \mathrm{m} 38.5$.

Preparation of RNA and northern analysis. MEFs were infected at an $\mathrm{MOI}$ of 5 . Infections were performed in the presence of either cyclohexamide (Sigma-Aldrich) $(50 \mu \mathrm{g} / \mathrm{ml})$ for immediate early RNA or phosphonoacetic acid (Sigma-Aldrich) $(20 \mu \mathrm{g} / \mathrm{ml})$ for early RNA, and isolation performed at $4 \mathrm{~h}$ after infection. For the collection of late RNA transcripts, MEFs were infected in the absence of metabolic inhibitors and harvested $24 \mathrm{~h}$ after infection. All RNA extractions were performed using Trizo $^{\mathrm{TM}}$ (Invitrogen) according to the manufacturer's instructions. RNA samples were subjected to electrophoresis under denaturing conditions, blotted onto a PVDF nylon membrane (Amersham Pharmacia) and hybridised with $\mathrm{P}^{32} \mathrm{dCTP}$-labelled double-stranded DNA probes. Probes for M38, m38.5, M39 and IE1 were radioactively labelled using the DECAprime $^{\mathrm{TM}}$ II labelling kit (Ambion) according to the manufacturer's instructions.

$3^{\prime}$ and $5^{\prime}$ rapid amplification of cDNA ends. $3^{\prime}$ and $5^{\prime}$ RACE PCR was performed using total RNA prepared from infected MEFs at immediate early, early and late times Pl using the FirstChoice RLM-RACE kit (Ambion) and m38.5- or M38specific oligonucleotides. Reactions were carried out according to the manufacturer's instructions. The cDNAs obtained were cloned into pGEMT-Easy and sequenced.

Construction of $\Delta \mathrm{m} 38.5 \mathrm{Rev}$. The m38.5 revertant virus (m38.5 Rev) was generated by co-transfecting MEF with $\triangle 38.5$ viral DNA and a pBluescript construct encoding the full-length m38.5 ORF. Virus stocks isolated from the co-transfection were used to infect MEF monolayers and, 4 days later, DNA was prepared using the Puregene genomic DNA purification kit (Gentra Systems). The presence of $\mathrm{m} 38.5$ revertant virus was detected by PCR and the $\mathrm{m} 38.5$ Rev virus was cloned by limiting dilution. Briefly, MEF monolayers in 24-well plates were infected with virus stocks from the co-transfection containing the m38.5 Rev virus at an MOI of 1 PFU per well and incubated until a cytopathic effect was evident. Viral supernatants were retained and DNA prepared from the infected cells. PCR was performed to identify wells containing $\Delta \mathrm{m} 38.5 \mathrm{Rev}$. 
Statistical analysis. All plotted data represent mean \pm 1 standard deviation. All $P$-values were determined using the non-parametric Mann-Whitney statistical test.

Acknowledgements. We are grateful to Dr. A Redwood for providing the K181 MCMV BAC. We thank Professor A Strasser and Associate Professor W Langdon for providing helpful insights during the preparation of the manuscript. This project was supported by grants from the National Health and Medical Research Council of Australia (NH\&MRC) and a Wellcome Trust (Overseas Senior Research Fellowship in Biomedical Science to MADE).

1. Boya P, Pauleau AL, Poncet D, Gonzalez-Polo RA, Zamzami N, Kroemer G. Viral proteins targeting mitochondria: controlling cell death. Biochim Biophys Acta 2004; 1659: 178-189.

2. Adams JM, Cory S. Bcl-2-regulated apoptosis: mechanism and therapeutic potential. Curr Opin Immunol 2007; 19: 488-496.

3. Lindsten T, Ross AJ, King A, Zong WX, Rathmell JC, Shiels HA et al. The combined functions of proapoptotic Bcl-2 family members Bak and Bax are essential for normal development of multiple tissues. Mol Cell 2000; 6: 1389-1399.

4. Cheng E, Wei MC, Weiler S, Flavell RA, Mak TW, Lindsten T et al. BCL-2, BCL-X-L sequester $\mathrm{BH} 3$ domain-only molecules preventing BAX-and BAK-mediated mitochondrial apoptosis. Mol Cell 2001; 8: 705-711.

5. Willis SN, Chen L, Dewson G, Wei A, Naik E, Fletcher $\mathrm{Jl}$ et al. Proapoptotic Bak is sequestered by $\mathrm{Mcl}-1$ and $\mathrm{Bcl}-\mathrm{xL}$, but not $\mathrm{Bcl}-2$, until displaced by $\mathrm{BH}$-only proteins. Genes Dev 2005; 19: 1294-1305

6. Willis SN, Fletcher Jl, Kaufmann T, van Delft MF, Chen L, Czabotar PE et al. Apoptosis initiated when BH3 ligands engage multiple Bcl-2 homologs, not Bax or Bak. Science 2007; 315: 856-859.

7. Uren RT, Dewson G, Chen L, Coyne SC, Huang DC, Adams JM et al. Mitochondrial permeabilization relies on $\mathrm{BH} 3$ ligands engaging multiple prosurvival $\mathrm{Bcl}-2$ relatives, not Bak. J Cell Biol 2007; 177: 277-287.

8. Knudson CM, Tung KS, Tourtellotte WG, Brown GA, Korsmeyer SJ. Bax-deficient mice with lymphoid hyperplasia and male germ cell death. Science 1995; 270: 96-99.

9. Cartron PF, Juin P, Oliver L, Martin S, Meflah K, Vallette FM. Nonredundant role of Bax and Bak in Bid-mediated apoptosis. Mol Cell Biol 2003; 23: 4701-4712

10. Andoniou CE, Degli-Esposti MA. Insights into the mechanisms of CMV-mediated interference with cellular apoptosis. Immunol Cell Biol 2006; 84: 99-106.

11. Andoniou CE, Andrews DM, Manzur M, Ricciardi-Castagnoli P, Degli-Esposti MA. A novel checkpoint in the $\mathrm{Bcl}-2$-regulated apoptotic pathway revealed by murine cytomegalovirus infection of dendritic cells. J Cell Biol 2004; 166: 827-837.

12. Poncet $D$, Larochette N, Pauleau AL, Boya P, Jalil AA, Cartron PF et al. An anti-apoptotic viral protein that recruits Bax to mitochondria. J Biol Chem 2004; 279: 22605-22614.

13. Arnoult D, Bartle LM, Skaletskaya A, Poncet D, Zamzami N, Park PU et al. Cytomegalovirus cell death suppressor VMIA blocks Bax- but not Bak-mediated apoptosis by binding and sequestering Bax at mitochondria. Proc Natl Acad Sci USA 2004: 101: 7988-7993.

14. Reboredo M, Greaves RF, Hahn G. Human cytomegalovirus proteins encoded by UL37 exon 1 protect infected fibroblasts against virus-induced apoptosis and are required for efficient virus replication. J Gen Virol 2004; 85: 3555-3567.

15. McCormick AL, Meiering CD, Smith GB, Mocarski ES. Mitochondrial cell death suppressors carried by human and murine cytomegalovirus confer resistance to proteasome inhibitor-induced apoptosis. J Virol 2005; 79: 12205-12217.

16. Murphy E, Yu D, Grimwood J, Schmutz J, Dickson M, Jarvis MA et al. Coding potential of laboratory and clinical strains of human cytomegalovirus. Proc Natl Acad Sci USA 2003; 100: $14976-14981$

17. Redwood AJ, Messerle M, Harvey NL, Hardy CM, Koszinowski UH, Lawson MA et al. Use of a murine cytomegalovirus K181-derived bacterial artificial chromosome as a vaccine vector for immunocontraception. J Virol 2005; 79: 2998-3008.
18. Henson D, Strano AJ. Mouse cytomegalovirus. Necrosis of infected and morphologically normal submaxillary gland acinar cells during termination of chronic infection. Am J Pathol 1972; 68: 183-202.

19. Stoddart CA, Cardin RD, Boname JM, Manning WC, Abenes GB, Mocarski ES. Peripheral blood mononuclear phagocytes mediate dissemination of murine cytomegalovirus. J Virol 1994; 68: 6243-6253.

20. Saederup N, Lin YC, Dairaghi DJ, Schall TJ, Mocarski ES. Cytomegalovirus-encoded beta chemokine promotes monocyte-associated viremia in the host. Proc Natl Acad Sci USA 1999; 96: 10881-10886.

21. Noda S, Aguirre SA, Bitmansour A, Brown JM, Sparer TE, Huang J et al. Cytomegalovirus MCK-2 controls mobilization and recruitment of myeloid progenitor cells to facilitate dissemination. Blood 2006; 107: 30-38

22. Benedict CA, De Trez C, Schneider K, Ha S, Patterson G, Ware CF. Specific remodeling of splenic architecture by cytomegalovirus. PLOS Pathog 2006; 2: e16.

23. Andrews DM, Andoniou CE, Granucci F, Ricciardi-Castagnoli P, Degli-Esposti MA. Infection of dendritic cells by murine cytomegalovirus induces functional paralysis. Nat Immunol 2001; 2: 1077-1084.

24. Perez D, White E. TNF-alpha signals apoptosis through a bid-dependent conformational change in Bax that is inhibited by E1B 19K. Mol Cell 2000; 6: 53-63.

25. Sundararajan R, White E. E1B 19K blocks Bax oligomerization and tumor necrosis factor alpha-mediated apoptosis. J Virol 2001; 75: 7506-7516.

26. Su J, Wang G, Barrett JW, Irvine TS, Gao X, McFadden G. Myxoma virus M11L blocks apoptosis through inhibition of conformational activation of Bax at the mitochondria. J Virol 2006; 80: 1140-1151.

27. Wang G, Barrett JW, Nazarian SH, Everett H, Gao X, Bleackley $\mathrm{C}$ et al. Myxoma virus M11L prevents apoptosis through constitutive interaction with Bak. J Virol 2004; 78 : 7097-7111.

28. Marshall WL, Yim C, Gustafson E, Graf T, Sage DR, Hanify K et al. Epstein-Barr virus encodes a novel homolog of the bcl-2 oncogene that inhibits apoptosis and associates with Bax and Bak. J Virol 1999; 73: 5181-5185.

29. Jurak I, Schumacher U, Simic H, Voigt S, Brune W. Murine cytomegalovirus m38.5 protein inhibits Bax-mediated cell death. J Virol 2008; 82: 4812-4822.

30. Norris KL, Youle RJ. Cytomegalovirus proteins vMIA and $\mathrm{m} 38.5$ link mitochondrial morphogenesis to Bcl-2 family proteins. J Virol 2008; 82: 6232-6243.

31. Arnoult D, Skaletskaya A, Estaquier J, Dufour C, Goldmacher VS. The murine cytomegalovirus cell death suppressor m38.5 binds Bax and blocks Bax-mediated mitochondrial outer membrane permeabilization. Apoptosis 2008; 13: 1100-1110.

32. Karbowski M, Norris KL, Cleland MM, Jeong SY, Youle RJ. Role of Bax and Bak in mitochondrial morphogenesis. Nature 2006; 443: 658-662.

33. Jones RG, Bui T, White C, Madesh M, Krawczyk CM, Lindsten T et al. The proapoptotic factors Bax and Bak regulate $T$ Cell proliferation through control of endoplasmic reticulum $\mathrm{Ca}(2+)$ homeostasis. Immunity 2007; 27: 268-280.

34. McCormick AL, Smith VL, Chow D, Mocarski ES. Disruption of mitochondrial networks by the human cytomegalovirus UL37 gene product viral mitochondrial-localized inhibitor of apopotsis. J Virol 2003; 77: 631-641.

35. Sharon-Friling R, Goodhouse J, Colberg-Poley AM, Shenk T. Human cytomegalovirus pUL37X1 induces the release of endoplasmic reticulum calcium stores. Proc Natl Acad Sc USA 2006; 103: 19117-19122.

36. Rowe WP, Hartley JW, Cramblett HG, Mastrota FM. Detection of human salivary gland virus in the mouth and urine of children. Am J Hyg 1958; 67: 57-65.

37. Krajewski S, Krajewska M, Shabaik A, Miyashita T, Wang HG, Reed JC. Immunohistochemical determination of in vivo distribution of Bax, a dominant inhibitor of Bcl-2. Am J Pathol 1994; 145: 1323-1336.

38. Chen L, Willis SN, Wei A, Smith BJ, Fletcher Jl, Hinds MG et al. Differential targeting of prosurvival $\mathrm{Bcl}-2$ proteins by their $\mathrm{BH} 3$-only ligands allows complementary apoptotic function. Mol Cell 2005; 17: 393-403.

39. Andoniou CE, van Dommelen SL, Voigt V, Andrews DM, Brizard G, Asselin-Paturel C et al. Interaction between conventional dendritic cells and natural killer cells is integral to the activation of effective antiviral immunity. Nat Immunol 2005; 6: 1011-1019.

40. Atalay R, Zimmermann A, Wagner M, Borst E, Benz C, Messerle M et al. Identification and expression of human cytomegalovirus transcription units coding for two distinct Fcgamma receptor homologs. J Virol 2002; 76: 8596-8608.

\section{Supplementary Information accompanies the paper on Cell Death and Differentiation website (http://www.nature.com/cdd)}

